\title{
ON THE ASYMPTOTIC MEMBRANE THEORY OF THIN HYPERELASTIC PLATES
}

\author{
H. A. ERBAY \\ Department of Mathematics, Faculty of Science, Istanbul Technical University, Maslak 80626, \\ Istanbul, Turkey
}

\begin{abstract}
Applying the asymptotic expansion technique to the three-dimensional equations of non-linear elasticity, a non-linear asymptotic membrane theory considering large deflections and strains is obtained for thin hyperelastic plates. To this end, the displacement vector and stress tensor components are scaled via an appropriate thickness parameter such that the present approximation takes into account larger deflections compared with those of the von Kármán plate theory. Later, for an arbitrary form of the strain energy function, the hierarchy of the field equations is obtained by expanding the displacement vector and the stress tensor in terms of powers of the square root of the thickness parameter. The equations belonging to the first three orders of this hierarchy are studied in detail. It is shown that the zeroth order approximation corresponds to the well-known Föppl membrane theory, the first order approximation includes bending effects, and the effect of material non-linearity appears in the second order approximation. Solving the problem of an infinitely long strip under uniform load for clamped edge conditions, the effect of material non-linearity is discussed numerically for both compressible and incompressible hyperelastic solids. The results are also compared with the solutions of the asymptotic approximation which gives the von Kármán plate equations in the zeroth order approximation. Copyright (C) 1997 Elsevier Science Ltd
\end{abstract}

\section{INTRODUCTION}

The aim of the present work is to construct an asymptotic non-linear membrane theory of thin elastic plates for general hyperelastic constitutive models and, then, is to discuss the effect of material non-linearity through a simple example.

In recent years, there have been many studies deducing two-dimensional non-linear plate theories from the original three-dimensional elasticity theory via asymptotic analysis. In these studies, the non-linear three-dimensional theory is expanded asymptotically with respect to a sufficiently small thickness parameter $\epsilon$, defined as the ratio of the plate thickness to a characteristic lateral dimension of the plate, and a two-dimensional non-linear mathematical model of plate is obtained as the leading term of the formal asymptotic expansion. Whereas some of the authors apply the asymptotic expansion method to the equations defined in variational form, others prefer to apply it directly to the field equations in local form. Since the questions of uniqueness, existence and convergence etc. are not to be dealt with, the second approach will be used in the present study, which seems more appropriate to obtain higher order equations in the hierarchy.

Employing the asymptotic expansion method, Habib [1], Ciarlet and Destuynder [2], Ciarlet [3], Suhubi [4], Coutris and Monavon [5] and Berg [6] obained the von Kármán plate equations [7] corresponding to geometrically non-linear, but materially linear, equations of elasticity. The first attempts to derive an asymptotic non-linear plate theory taking into account material (i.e. physical) non-linearity are made by Johnson and Urbanik [8] and Johnson [9]. But, in these works the non-linear constitutive equations are approximated by an assumption on the nature of the complementary energy function to avoid an inconsistent theory. Later, following the asymptotic approach of [2] and [3] which use a variational formulation, Davet [10] shows that the effect of material non-linearity does not appear in the zeroth order approximation, i.e. the zeroth order equations for any non-linear constitutive law is always the same. Recently, using a reformulation of the asymptotic procedure introduced in [2] and [3], Karwowski [11] and Fox 
et al. [12] reach the same conclusion. Furthermore, they also prove that the non-linear membrane model arising as the leading-order term of the asymptotic analysis for a different scaling does not depend on the particular choice of the constitutive law. However, these works do not present the higher order equations in which the effect of material non-linearity appears.

In [13], a non-linear theory for thin hyperelastic plates is developed by Erbay and Suhubi applying the asymptotic expansion method directly to the field equations in local form. Confirming the result in [10], it is shown that the effect of material non-linearity does not appear in the zeroth order approximation (which corresponds to the von Kármán plate theory) and to include this effect one must proceed to higher order approximations. In addition, the first order equations including this effect are given explicitly, which, as is expected, are more complex than those in the zeroth order approximation. In [14], this approximation is extended to incompressible hyperelastic solids by the same authors. It is shown that, using a transformation between the coefficients in both cases, it is possible to obtain the equations for incompressible hyperelastic plates from those for compressible ones. As a simple application of the theory, the problem of an infinitely long strip subject to uniform load is also investigated and the results corresponding to the materially linear and non-linear elastic solids are compared numerically. It is pointed out that, for the effect of material non-lineaity to be significant, very large deflections compared to the thickness have to be considered. Moreover, it is demonstrated that as deflections increase the solutions are affected relatively less by the boundary conditions and that, for the deflections in which the effect of material non-linearity becomes significant, the membrane effects dominate everywhere except for the region near the edge. Similar remarks are also made by Brodland [15] for the problem of highly non-linear deformation of uniformly-loaded circular plates, where a numerical solution based on a relaxed Kirchhoff hypothesis is used.

The present study which derives an asymptotic non-linear membrane theory taking into account material non-linearity is based on the above-mentioned observations and the techniques developed in [13]. It takes into account larger deflections compared to the von Kármán plate theory and gives more importance to the membrane effects. In Section 2 , the three-dimensional field equations for non-linear elastic solids are stated and the plate geometry and the dimensionless coordinates are defined. Later, utilizing the thickness parameter, the displacement vector and the stress tensor components are scaled such that the above observations are taken into account.

In Section 3, the displacement vector and the stress tensor are expanded in powers of $\epsilon^{1 / 2}$ and the hierarchies of equilibrium equations and boundary conditions are obtained. Similarly, the hierarchy of constitutive equations corresponding to non-linear isotropic elastic solids is found for an arbitrary form of the strain energy function. The equations corresponding to the zeroth, first and second order approximations are studied in detail. It is seen that the zeroth order approximation corresponds to the well-known Föppl membrane theory $[7,16]$, the first order approximation includes the bending effects, and the effect of material non-linearity appears only in the second order approximation. For the lowest three order approximations, it is possible to decompose the total stress into bending and membrane stress components. It is also pointed out that the results obtained are also valid for incompressible hyperelastic plates with the use of a transformation between the material coefficients.

In Section 4, the problem of an infinite strip subject to uniform load, which has been studied in [14], is solved analytically using the present approach. The results in the special cases of the Ko and neo-Hookean solids which are the simplest representative examples of compressible and incompressible hyperelastic materials, respectively are compared with the solutions based only upon the geometric non-linearity. A similar comparison is also made with the results of [14]. The last section is devoted to conclusions. 


\section{THE THREE-DIMENSIONAL PROBLEM AND SCALING}

\subsection{Notation}

Assume that in the reference configuration a plate of uniform thickness $2 h$ occupies a domain $R=\Omega \times(-h, h)$ where $\Omega$ shows the middle plane of the plate and is an open set with a sufficiently smooth boundary $C$. The upper, lower and lateral surfaces of the plate will be denoted by $\Omega^{+}=\Omega \times\{h\}, \Omega^{-}=\Omega \times\{-h\}$ and $\Sigma=C \times[-h, h]$, respectively. The curvilinear material coordinates $X^{K}$ are so chosen that $X^{1}$ and $X^{2}$ curves are plane curves parallel to $\Omega$ and $X^{3}$ is perpendicular to $\Omega$ which is the plane $X^{3}=0$. Thus the components of metric tensor $G_{K L}$ become $G_{\alpha \beta}\left(X^{\gamma}\right)=A_{\alpha \beta}\left(X^{\gamma}\right), G_{\alpha 3}=0$ and $G_{33}=1$ where $A_{\alpha \beta}$ is the metric tensor of the middle planet. The surface tractions prescribed on the upper and lower faces are shown with $\mathbf{t}_{+}$and $\mathbf{t}_{-}$, respectively and the stress vector on the lateral surface by $\mathbf{t}$.

\subsection{Three-dimensional equations}

The equilibrium equations and the boundary conditions in the reference configuration can be written as follows $[13,17]$ :

$$
\begin{gathered}
\left(T^{K L}+T^{K M} U_{; M}^{L}\right)_{; K}+\rho_{0} F^{L}=0 \quad \text { in } R \\
T^{3 L}+T^{3 M} U_{; M}^{L}=\mp t_{\mp}^{L} \text { on } \Omega^{\mp} \\
\left(T^{\alpha L}+T^{\alpha M} U_{; M}^{L}\right) N_{\alpha}=\bar{t}^{L} \quad \text { on } \Sigma
\end{gathered}
$$

where $T^{K L}$ is the second Piola-Kirchhoff stress tensor, $U^{K}$ is the displacement vector, $\rho_{0}$ is the initial density, $F^{L}$ is the body force per unit mass, $N_{\alpha}$ is the unit exterior normal to $\Sigma$ and a semi-colon denotes the partial covariant differentiation. As it is known, in order to achieve a state of membrane stress, the edges of the plate must not be subject to transverse shear forces or bending moments. In other words, the plate in the membrane approximation must carry only tangential loads at its edge and in this case one cannot take into account all the three-dimensional boundary conditions. However, for the sake of completeness the exact three-dimensional boundary conditions are given in equation (2.3). Sometimes it may be more reasonable to prescribe the boundary conditions on the displacement components on a part or total of $\Sigma$. In such a case the boundary conditions (2.3) must be replaced with those depending on the displacement components only. For example, the boundary conditions for clamped (i.e. fixed) edges are written as $U^{K}=0$ on $\Sigma$. The Lagrangian strain tensor $E_{K L}$ and the basic invariants of $E_{K L}$ are defined by

$$
2 E_{K L}=U_{K ; L}+U_{L ; K}+U_{M ; K} U_{; L}^{M}
$$

and

$$
I_{E}=\operatorname{tr} \mathbf{E}, \quad I I_{E}=\frac{1}{2}\left[(\operatorname{tr} \mathbf{E})^{2}-\operatorname{tr} \mathbf{E}^{2}\right], \quad I I I_{E}=\frac{1}{3}\left[\operatorname{tr} \mathbf{E}^{3}-\frac{3}{2}(\operatorname{tr} \mathbf{E})\left(\operatorname{tr} \mathbf{E}^{2}\right)+\frac{1}{2}(\operatorname{tr} \mathbf{E})^{3}\right]
$$

respectively. Also the constitutive equations of homogeneous isotropic hyperelastic solids are

$$
T^{K L}=\tilde{e}_{1} G^{K L}+\tilde{e}_{2} E^{K L}+\tilde{e}_{3} E^{K}{ }_{M} E^{M L}
$$

where the coefficients $\tilde{e}_{k}(k=1,2,3)$ are in the following form:

$$
\tilde{e}_{1}=\frac{\partial \tilde{\Sigma}}{\partial I_{E}}+I_{E} \frac{\partial \tilde{\Sigma}}{\partial I I_{E}}+I I_{E} \frac{\partial \tilde{\Sigma}}{\partial I I I_{E}}, \quad \tilde{e}_{2}=-\left(\frac{\partial \tilde{\Sigma}}{\partial I I_{E}}+I_{E} \frac{\partial \tilde{\Sigma}}{\partial I I I_{E}}\right), \quad \tilde{e}_{3}=\frac{\partial \tilde{\Sigma}}{\partial I I I_{E}}
$$


Here $\tilde{\Sigma}\left(I_{E}, I I_{E}, I I I_{E}\right)$ is the strain energy function. If the solid in the reference configuration is assumed to be stress free, then the following restriction on $\tilde{\Sigma}$ is satisfied:

$$
\frac{\partial \tilde{\Sigma}}{\partial I_{E}}=0 \quad \text { when } \quad I_{E}=I I_{E}=I I I_{E}=0 \text {. }
$$

\subsection{Scaling}

The thickness parameter $\epsilon$ (sometimes it is called geometric parameter) is defined in the form $\epsilon=h / L$ where $2 L$ is a characteristic lateral length scale. The thin plate assumption implies that $\epsilon \ll 1$. Now the following dimensionless coordinates are introduced:

$$
X^{\alpha}=L \xi^{\alpha}, \quad X^{3}=\epsilon L \xi^{3}, \quad \xi^{3} \in[-1,1]
$$

which transform the domain occupied by the thin plate into a domain of comparable dimensions, which is independent of $\epsilon$. The upper, lower, and lateral surfaces of the plate in the dimensionless coordinates are denoted by $\omega^{+}, \omega^{-}$, and $\sigma$, respectively and the middle plane and its boundary by $\omega$ and $c$. Since such a transformation introduces the parameter $\epsilon$ into the field equations, the solution of the three-dimensional boundary value problem defined in equations (2.1)-(2.8) will depend not only on $\xi$ but also on $\epsilon$. It is reasonable then to expect the solution of the corresponding boundary-value problem to have an expansion with respect to $\epsilon$. In what follows, the displacement vector and stress tensor components in the dimensionless coordinates will be scaled by some particular powers of $\epsilon$ such that it reflects the expected behaviour of the plate and then a consistent asymptotic membrane theory for thin hyperelastic plates will be developed without any additional assumptions. But, at this point, note that the asymptotic expansion method is not free from a priori assumptions due to the scaling assertions. Now the non-dimensional displacement vector and stress tensor components in $\xi$-coordinates are defined in the following form:

$$
\begin{gathered}
U^{\alpha}(\mathbf{X})=\epsilon L u^{\alpha}(\xi), \quad U^{3}(\mathbf{X})=\epsilon^{\frac{1}{2}} L u^{3}(\xi) \\
T^{\alpha \beta}(\mathbf{X})=T_{0} \epsilon \sigma^{\alpha \beta}(\xi), \quad T^{\alpha 3}(\mathbf{X})=T_{0} \epsilon^{2} \sigma^{\alpha 3}(\xi), \quad T^{33}(\mathbf{X})=T_{0} \epsilon^{\frac{5}{2}} \sigma^{33}(\xi)
\end{gathered}
$$

where $T_{0}$ is an appropriately chosen factor of stress dimension. As is seen from equation (2.10), the transverse displacement component $U^{3}$ is assumed to be small compared to the plane dimensions of the plate, but may be large compared to the thickness. A comparison of this scaling with that corresponding to the von Kármán plate theory, which is given in [13], shows that the present approximation considers larger transverse deffections compared to the von Kármán theory where the deflections are assumed to be at the order of $\epsilon$ (or the thickness). On the other hand the scale of the lateral displacement components $U^{\alpha}$ is chosen such that the zeroth order displacement field is free from the transverse coordinate $\xi^{3}$, i.e. it is a membrane displacement field. In other words, the scale of $U^{\alpha}$ is determined so as to ensure that the transverse shear components of the strain tensor are zero at the zeroth order approximation. Moreover, the scaling of planar components of the strain tensor implies the scaling of in-plane components of the stress tensor. If $\epsilon^{\frac{1}{2}}$ is considered as a perturbation parameter, it is seen from (2.10) that the transverse components with respect to in-plane components of the stress tensor are shifted to higher orders compared to von Kármán theory [13] and from the point of view of the membrane approach the present approximation seems physically reasonable. Finally, note that the scaling in equation (2.10) corresponds to the special cases of the scalings given for the strong loading case in Table 1 of [5] and given for the membrane model considering moderately large displacements in [11].

The non-dimensional surface and body force densities are defined by

$$
\begin{gathered}
t_{\mp}^{\alpha}\left(X^{\beta}\right)=T_{0} \epsilon^{2} g_{\mp}^{\alpha}\left(\xi^{\beta}\right), \quad t_{\mp}^{3}\left(X^{\beta}\right)=T_{0} \epsilon^{\frac{5}{2}} g_{\mp}^{3}\left(\xi^{\beta}\right), \quad \mathbf{X} \in \Omega^{\mp}, \quad \xi \in \omega^{\mp} \\
\bar{t}^{\alpha}(\mathbf{X})=T_{0} \epsilon \tau^{\alpha}(\xi), \quad \bar{t}^{3}(\mathbf{X})=T_{0} \epsilon^{\frac{3}{2}} \tau^{3}(\xi), \quad \mathbf{X} \in C, \quad \xi \in c \\
\rho_{0} L F^{\alpha}(\mathbf{X})=T_{0} \epsilon f^{\alpha}(\xi), \quad \rho_{0} L F^{3}(\mathbf{X})=T_{0} \epsilon^{\frac{3}{2}} f^{3}(\boldsymbol{\xi}) .
\end{gathered}
$$


Here the above quantities are scaled such that the non-dimensional surface and body force densities are included in the lowest order approximation and external loads tend to zero with the vanishing thickness. An asymptotic expansion based on the above equations will be constructed in the following section.

\section{ASYMPTOTIC EXPANSION}

\subsection{Asymptotic expansion}

As it is mentioned in the previous section, the solution of the three-dimensional boundaryvalue problem defined in the dimensionless coordinates will depend not only on $\xi$, but also on $\epsilon$. Now, recalling the dependence of the equations written in the dimensionless coordinates on $\epsilon^{\frac{1}{2}}$, it is assumed that the displacement vector $\mathbf{u}$ and the stress tensor $\sigma$ may be expanded in an asymptotic power series as follows:

$$
\mathbf{u}(\boldsymbol{\xi})=\sum_{n=0}^{\infty} \delta^{n} \mathbf{u}(\boldsymbol{\xi}), \quad \boldsymbol{\sigma}(\boldsymbol{\xi})=\sum_{n=0}^{\infty} \delta^{n} \stackrel{(n)}{\boldsymbol{\sigma}}(\boldsymbol{\xi})
$$

where the definition $\delta=\epsilon^{\frac{1}{2}}$ is used for convenience. As can be seen from (2.10) and (2.11), displacements, stresses and external loads tend to zero as $\delta$ tends to zero. In other words, (3.1) is an asymptotic expansion about the natural (or undeformed) state. The scaling made in equations (2.10)-(2.11) and the expansion (3.1) are the main assumptions of this paper and an asymptotic theory will be developed without any additional assumptions. It is expected that the first few terms of this expansion will identify the main features of the true solution of the three-dimensional boundary-value problem. In this section, the equations corresponding to these first few terms will be derived and a correspondence between the equations of two- and three-dimensional problems will be established.

\subsection{Hierarchies of basic equations}

Now, using the asymptotic expansion (3.1), the hierarchies of the strain-displacement relations, the equilibrium equations and the boundary conditions will be obtained respectively. To this end, substituting (3.1) $)_{1}$ into (2.4), the following expansion is obtained for the Lagrange strain tensor:

$$
\left\{E_{\alpha \beta}, E_{\alpha 3}, E_{33}\right\}=\sum_{n=0}^{\infty}\left\{\delta^{2} \stackrel{(n)}{E}_{\alpha \beta}, \delta \stackrel{(n)}{E}_{\alpha 3}, \delta \stackrel{(n)}{E}_{33}\right\} \delta^{n}
$$

where

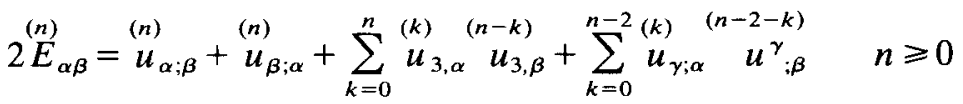

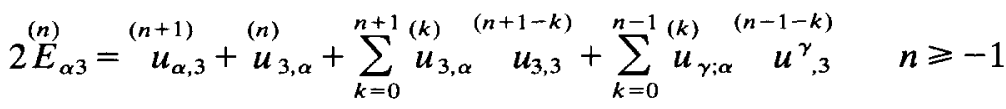

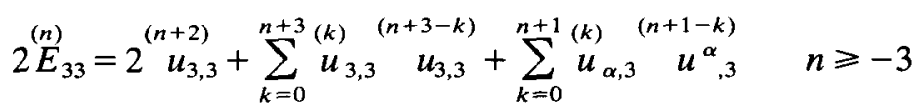

in which derivatives are taken with respect to $\xi$-coordinates. Since the Lagrange strain tensor components should tend to zero as $\delta$ tends to zero, the components having negative values of $n$ in these expressions should vanish. This imposes some constraints on the displacement field,

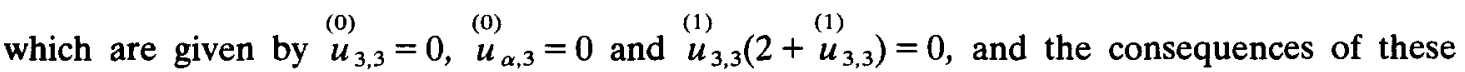
constraints will be discussed in detail later. Similarly, substituting (3.2) into (2.5) it is found that

$$
\left\{I_{E}, I I_{E}, I I I_{E}\right\}=\sum_{n=0}^{\infty}\left\{\delta \stackrel{(n)}{I}_{E}, \delta^{2} \stackrel{(n)}{I I} I_{E}, \delta^{4} I I I_{E}\right\} \delta^{n}
$$


where, for $n \geqslant 0$,

$$
\begin{aligned}
& \stackrel{(n)}{I_{E}}=\stackrel{(n-1)}{E_{\alpha}^{\alpha}}+\stackrel{(n)}{E_{3}^{3}}
\end{aligned}
$$

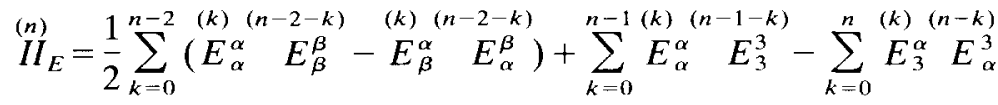

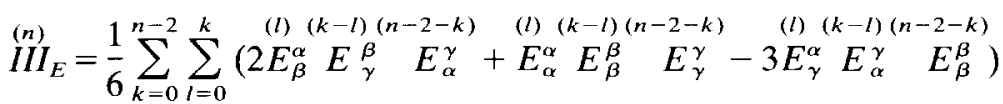

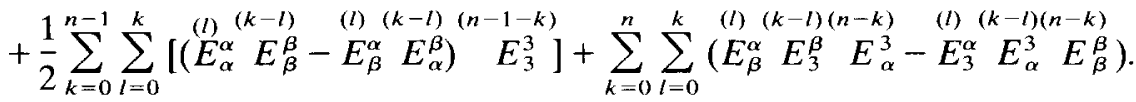

From now on, the quantities having negative index will be taken as zero.

In order to obtain the hierarchy of the equilibrium equations, (3.1) is employed in (2.1) and the result is

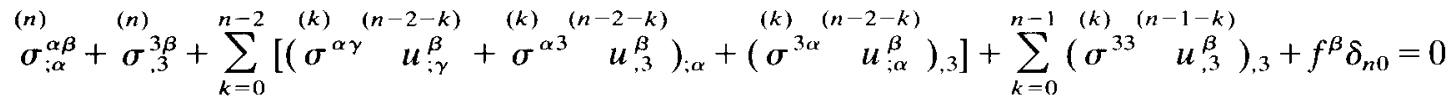

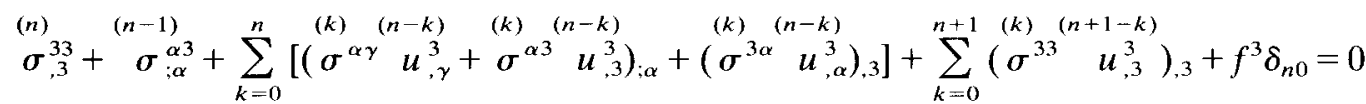

for $n \geqslant 0$, where $\delta_{m n}$ is the Kronecker symbol. With a similar process the boundary conditions (2.2)-(2.3) take the following form:

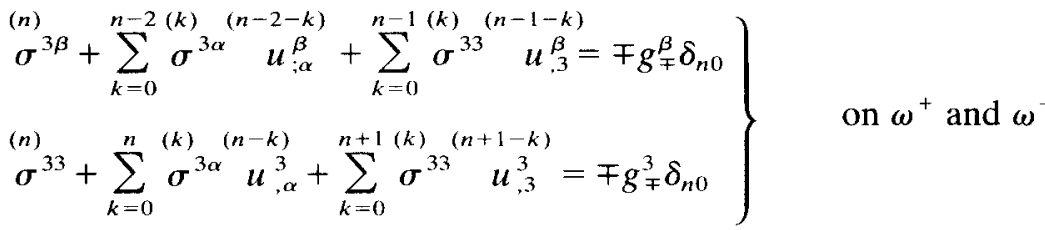

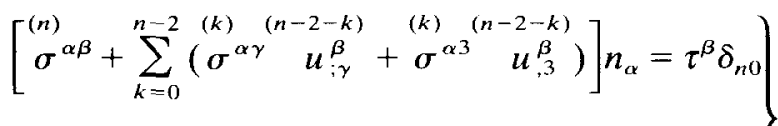

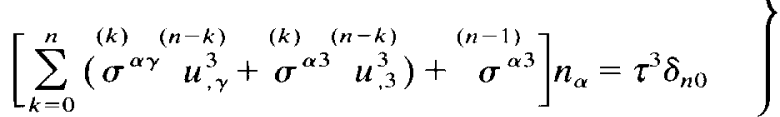

for $n \geqslant 0$, where $n_{\alpha}$ is the exterior unit normal to $\sigma$. On the other hand, the boundary conditions for clamped edge can be expressed as $\stackrel{(n)}{u^{\alpha}}=0$ and $\stackrel{(n)}{u^{3}}=0$ on $\sigma$ for $n \geqslant 0$.

\subsection{Hierarchy of constitutive equations}

In order to find the hierarchy of the constitutive equations, first the following expansion for the coefficients $\tilde{e}_{k}(k=1,2,3)$ appearing in the constitutive relations (2.6) is assumed:

$$
e_{k}=\sum_{n=0}^{\infty} \stackrel{(n)}{e}_{k} \delta^{n} \quad(k=1,2,3)
$$

where the definition $e_{k}=\tilde{e}_{k} / T_{0}$ is used. The explicit forms of the quantities $\stackrel{(n)}{e_{k}}$ will be later calculated from the definitions (2.7) utilizing Taylor series of an arbitrary function of the invariants about $\delta=0$. For the present the explicit expressions of $\stackrel{(0)}{e_{k}}(k=1,2,3)$ can be easily calculated from (2.7) as follows:

$$
\stackrel{(0)}{e_{1}}=\left.\frac{\partial \Sigma}{\partial I_{E}}\right|_{0}, \quad \stackrel{(0)}{e_{2}}=-\left.\frac{\partial \Sigma}{\partial I I_{E}}\right|_{0}, \quad \stackrel{(0)}{e_{3}}=\left.\frac{\partial \Sigma}{\partial I I I_{E}}\right|_{0}
$$


where $\Sigma=\tilde{\Sigma} / T_{0}$ and the subscript 0 represents the case where the quantities are evaluated at $\delta=0$ and consequently at $I_{E}=I I_{E}=I I I_{E}=0$. Then, introducing equations (3.1), (3.2) and (3.9) into equation (2.6) the hierarchy of the constitutive relations is found in the form

$$
\begin{aligned}
& \stackrel{(n)}{\sigma^{\alpha \beta}}=\stackrel{(n+2)}{e_{1}} A^{\alpha \beta}+\sum_{k=0}^{n} \stackrel{(k)}{e}_{2}^{(n-k)} E^{\alpha \beta}+\sum_{k=0}^{n-2} \sum_{l=0}^{k} \stackrel{(l)}{e}_{3} E_{\gamma}^{(k-l)(n-2-k)} E^{\gamma \beta}+\sum_{k=0}^{n} \sum_{l=0}^{k} \stackrel{(l)}{e}_{3}^{(k-l)(n-k)} E_{3}^{\alpha} E^{3 \beta} \quad n \geqslant-2
\end{aligned}
$$

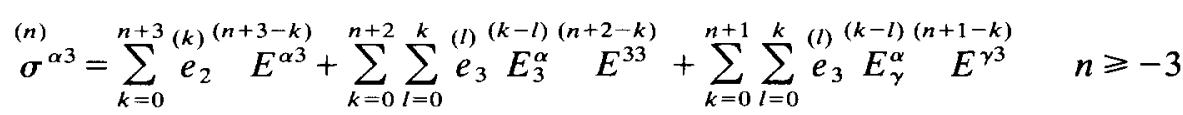

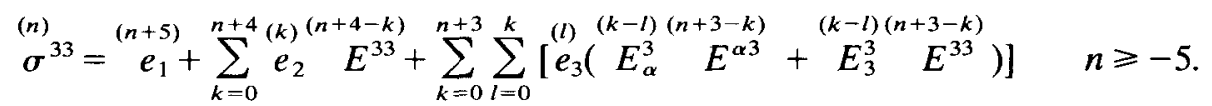

Since the expansion $(3.1)_{2}$ suggests that the stress components having negative index are zero, some constraints on the strain tensor components and on the coefficients $\stackrel{(n)}{e_{k}}$ are obtained. Now these constraints will be discussed below respectively. The most dominant restrictions are

$$
\begin{aligned}
& \stackrel{(-2)}{\sigma^{\alpha \beta}}=\stackrel{(-5)}{\sigma^{33}}=0 \Rightarrow \stackrel{(0)}{e_{1}}=0 \quad \text { or }\left.\quad \frac{\partial \Sigma}{\partial I_{E}}\right|_{0}=0 \\
& \stackrel{(-3)}{\sigma^{\alpha 3}}=0 \Rightarrow \stackrel{(0)}{e_{2} E^{\alpha 3}}=0 \quad \text { or }\left.\quad \stackrel{(0)}{E^{\alpha 3}} \frac{\partial \Sigma}{\partial I I_{E}}\right|_{0}=0
\end{aligned}
$$

where (3.10) is used. Recalling from (3.4) that $I_{E}=I I_{E}=I I I_{E}=0$ at $\delta=0$, it is seen that the condition (3.12) is equivalent to (2.8). On the other hand, since $\Sigma$ is assumed to be an arbitrary function of the invariants and the resulting non-linear theory must include the linear theory, from equation (3.13) it follows that $\stackrel{(0)}{E^{\alpha 3}}=0$. Employing this result in (3.11), the following conditions are also written:

$$
\begin{gathered}
\stackrel{(-1)}{\sigma^{\alpha \beta}}=0 \Rightarrow \stackrel{(1)}{e_{1}}=0 \\
\stackrel{(-2)}{\sigma^{\alpha 3}}=0 \Rightarrow \stackrel{(0)}{e_{2} E^{\alpha 3}}=0 \quad \text { or } \quad \stackrel{(1)}{\left.E^{\alpha 3} \frac{\partial \Sigma}{\partial I I_{E}}\right|_{0}=0} \\
\stackrel{(-4)}{\sigma^{33}}=0 \Rightarrow \stackrel{(1)}{e_{1}}+\stackrel{(0)}{e_{2} E^{33}}=0 \quad \text { or } \quad \stackrel{(0)}{\left.E^{33} \frac{\partial \Sigma}{\partial I I_{E}}\right|_{0}=0 .}
\end{gathered}
$$

With a similar argument, the conditions (3.15) and (3.16) lead to the results $\stackrel{(1)}{E^{\alpha 3}}=0$ and $\stackrel{(0)}{E^{33}}=0$, respectively. Similarly the condition

$$
\stackrel{(-1)}{\sigma^{\alpha 3}}=0 \Rightarrow \stackrel{(0)}{e_{2} E^{\alpha 3}}=0 \quad \text { or }\left.\quad \stackrel{(2)}{E^{\alpha 3}} \frac{\partial \Sigma}{\partial I_{E}}\right|_{0}=0
$$
gives $\stackrel{(2)}{E^{\alpha 3}}=0$. Now the remaining constraints are written as follows:

$$
\begin{gathered}
\stackrel{(-3)}{\sigma^{33}}=0 \Rightarrow \stackrel{(2)}{e}_{1}+\stackrel{(0)}{e}_{2} \stackrel{(1)}{E}_{33}=0 \\
\stackrel{(-2)}{\sigma^{33}}=0 \Rightarrow \stackrel{(3)}{e_{1}}+\stackrel{(0)}{e}_{2} \stackrel{(2)}{E}_{33}+\stackrel{(1)}{e}_{2} E_{33}=0 \\
\stackrel{(-1)}{\sigma^{33}}=0 \Rightarrow \stackrel{(4)}{e}_{1}+\stackrel{(0)}{e}_{2} \stackrel{(3)}{E}_{33}+\stackrel{(1)}{e}_{2}^{(2)} \stackrel{(2)}{E}_{33}+\stackrel{(2)}{e}_{2} \stackrel{(1)}{E}_{33}+\stackrel{(0)}{e_{3}} \stackrel{(1)}{E}_{33} \stackrel{(1)}{E}_{33}=0 .
\end{gathered}
$$


After calculating the explicit forms of the coefficients $\stackrel{(n)}{e_{k}}$, these conditions will be investigated in detail. The presently obtained constraints on the strain tensor are written once again altogether:

$$
\stackrel{(0)}{E}_{33}=0, \quad \stackrel{(0)}{E}_{\alpha 3}=\stackrel{(1)}{E}_{\alpha 3}=\stackrel{(2)}{E}_{\alpha 3}=0 .
$$

Using these results in equation (3.5), the following restrictions on the invariants are found:

$$
\stackrel{(0)}{I}_{E}=0, \quad \stackrel{(0)}{I}_{E}=\stackrel{(1)}{I}_{E}=0, \quad \stackrel{(0)}{I I}_{E}=\stackrel{(1)}{I I}_{E}=0 .
$$

Recalling that the lowest three order approximations only are concerned with, the corresponding in-plane stress components can be written from equation (3.11) in the following form:

$$
\begin{aligned}
& \stackrel{(0)}{\sigma^{\alpha \beta}}=\stackrel{(2)}{e_{1}} A^{\alpha \beta}+\stackrel{(0)}{e}{ }_{2} E^{\alpha \beta} \\
& \stackrel{(1)}{\sigma^{\alpha \beta}}=\stackrel{(3)}{e} A_{1} A^{\alpha \beta}+\stackrel{(0)}{e_{2}} E^{\alpha \beta}+\stackrel{(1)}{e_{2}} E^{\alpha \beta} \\
& \stackrel{(2)}{\sigma^{\alpha \beta}}=\stackrel{(4)}{e_{1}} A^{\alpha \beta}+\stackrel{(0)}{e_{2}} E^{(2)}+\stackrel{(1)}{e_{2}} E^{\alpha \beta}+\stackrel{(2)}{e_{2}} E^{\alpha \beta}+\stackrel{(0)}{e_{3} E_{\gamma}^{\alpha} E^{\gamma \beta}} \text {. }
\end{aligned}
$$

In order to calculate the coefficients $\stackrel{(n)}{e_{k}}$ appearing in equation (3.23), the following Taylor expansion of an arbitrary function of the invariants about $\delta=0$ is used:

$$
\begin{aligned}
f\left(I_{E}, I I_{E}, I I I_{E}\right)=f(0,0,0)+\left.\stackrel{(1)}{I_{E}} \frac{\partial f}{\partial I_{E}}\right|_{0} \delta^{2} & +\left.\stackrel{(2)}{I_{E}} \frac{\partial f}{\partial I_{E}}\right|_{0} \delta^{3} \\
& +\left.\left[\stackrel{(3)}{I_{E}} \frac{\partial f}{\partial I_{E}}+\stackrel{(2)}{I} I_{E} \frac{\partial f}{\partial I_{E}}+\frac{1}{2}\left(\stackrel{(1)}{I_{E}}\right)^{2} \frac{\partial^{2} f}{\partial I_{E}^{2}}\right]\right|_{0} \delta^{4}+\cdots
\end{aligned}
$$

where (3.22) is utilized. Thus, employing equations (3.4), (3.9) and (3.24) in (2.7), the $(n)$ coefficients $e_{k}$ appearing in (3.23) are calculated in the form

$$
\begin{aligned}
& \stackrel{(0)}{e_{1}}=0, \quad \stackrel{(0)}{e_{2}}=\Gamma_{1}, \quad \stackrel{(0)}{e_{3}}=\Gamma_{2} \\
& \stackrel{(1)}{e_{1}}=\stackrel{(1)}{e_{2}}=\stackrel{(1)}{e_{3}}=0, \quad \stackrel{(2)}{e_{1}}=\Gamma_{0} \stackrel{(1)}{I_{E}}, \quad \stackrel{(2)}{e_{2}}=-\Gamma_{3} \stackrel{(1)}{I_{E}} \\
& \left.\stackrel{(3)}{e_{1}}=\Gamma_{0} \stackrel{(2)}{I_{E}}, \quad \stackrel{(3)}{e_{2}}=-\Gamma_{3}^{(2)}, \quad \stackrel{(4)}{I_{1}}=\Gamma_{0} \stackrel{(3)}{I_{E}}+\Gamma_{3} \stackrel{(2)}{I_{E}}+\Gamma_{4} \stackrel{(1)}{I_{E}}\right)^{2}
\end{aligned}
$$

where the constants $\Gamma_{k}(k=0,1,2,3,4)$ are given by

$$
\begin{aligned}
& \Gamma_{0}=\left.\left(\frac{\partial^{2} \Sigma}{\partial I_{E}^{2}}+\frac{\partial \Sigma}{\partial I I_{E}}\right)\right|_{0}, \quad \Gamma_{1}=-\left.\frac{\partial \Sigma}{\partial I I_{E}}\right|_{0}, \quad \Gamma_{2}=\left.\frac{\partial \Sigma}{\partial I I I_{E}}\right|_{0}, \\
& \Gamma_{3}=\left.\left(\frac{\partial^{2} \Sigma}{\partial I_{E} \partial I_{E}}+\frac{\partial \Sigma}{\partial I I_{E}}\right)\right|_{0}, \quad \Gamma_{4}=\left.\left(\frac{1}{2} \frac{\partial^{3} \Sigma}{\partial I_{E}^{3}}+\frac{\partial^{2} \Sigma}{\partial I_{E} \partial I_{E}}\right)\right|_{0}
\end{aligned}
$$

Note that these definitions are the same as those in the equations (3.20) of [13] and that the condition (3.14) is identically satisfied due to (3.25). Next step is to introduce (3.25) into the constitutive relations (3.23). But, $\stackrel{(2)}{e_{1}}, \stackrel{(3)}{e_{1}}$ and $\stackrel{(4)}{e_{1}}$ (and consequently, $\underset{\sigma}{\sigma \beta}, \stackrel{(1)}{\sigma^{\alpha \beta}}$ and $\stackrel{(2)}{\sigma^{\alpha \beta}}$ ) 
include $\stackrel{(1)}{E}_{33}, \stackrel{(2)}{E}_{33}$, and $\stackrel{(3)}{E}_{33}$, respectively. In order to reach a consistent asymptotic theory $\stackrel{(1)}{E}_{33}$, $\stackrel{(2)}{E}_{33}$ and $\stackrel{(3)}{E}_{33}$ (which contain the higher order displacement terms such as $\stackrel{(3)}{u_{3,3}}, \stackrel{(4)}{u_{3,3}}$ and $\stackrel{(5)}{u_{3,3}}$ ) must be eliminated from the stress components (3.23). Employing the restrictions (3.18)-(3.20), the following results are obtained:

$$
\begin{aligned}
& \stackrel{(-3)}{\sigma^{33}}=0 \Rightarrow \stackrel{(1)}{E}=-\frac{\Gamma_{0}}{\Gamma_{0}+\Gamma_{1}} \stackrel{(0)}{E}_{\gamma}^{\gamma} \\
& \stackrel{(-2)}{\sigma^{33}}=0 \Rightarrow \stackrel{(2)}{E}=-\frac{\Gamma_{0}}{\Gamma_{0}+\Gamma_{1}} \stackrel{(1)}{E}_{\gamma}^{\gamma} \\
& \stackrel{(-1)}{\sigma^{33}}=0 \Rightarrow \stackrel{(3)}{E}_{33}=-\frac{\Gamma_{0}}{\Gamma_{0}+\Gamma_{1}} \stackrel{(2)}{E}_{\gamma}^{\gamma}-\frac{1}{\Gamma_{0}+\Gamma_{1}}\left[\frac{1}{2} \Gamma_{3}\left(\stackrel{(0)}{E_{\gamma}^{\gamma}} \stackrel{(0)}{E}_{\delta}^{\delta}-\stackrel{(0)}{E_{\delta}^{\gamma}} \stackrel{(0)}{E}_{\gamma}^{\delta}\right)+\Gamma_{4} \stackrel{(0)}{E}_{\gamma}^{\gamma} \stackrel{(0)}{\delta}_{\delta}^{\delta}\right. \\
& \left.+2 \Gamma_{4} \stackrel{(0)}{E}_{\gamma}^{\gamma} \stackrel{(1)}{E}_{33}+\left(\Gamma_{4}-\Gamma_{3}+\Gamma_{2}\right) \stackrel{(1)}{E}_{33}^{(1)} E_{33}\right] \text {. }
\end{aligned}
$$

Using these results and equation (3.25) in equation (3.23) the reduced constitutive equations now can be written as

$$
\begin{aligned}
& \stackrel{(0)}{\sigma^{\alpha \beta}}=2 \Delta_{1}\left({\stackrel{(0)}{\Delta_{0}} E_{\gamma}^{\gamma}}^{\alpha \beta}+\stackrel{(0)}{E^{\alpha \beta}}\right) \\
& \stackrel{(1)}{\sigma^{\alpha \beta}}=2 \Delta_{1}\left(\stackrel{(1)}{0}_{0} E_{\gamma}^{\gamma} A^{\alpha \beta}+\stackrel{(1)}{E^{\alpha \beta}}\right)
\end{aligned}
$$

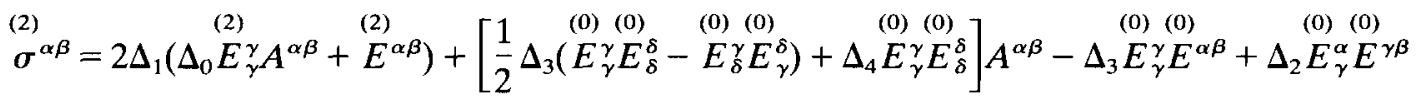

where the coefficients $\Delta_{k}(k=0,1,2,3,4)$ are defined by

$$
\begin{aligned}
& \Delta_{0}=\Gamma_{0} /\left(\Gamma_{0}+\Gamma_{1}\right), \quad \Delta_{1}=\Gamma_{1} / 2, \quad \Delta_{2}=\Gamma_{2}, \quad \Delta_{3}=\Gamma_{1} \Gamma_{3} /\left(\Gamma_{0}+\Gamma_{1}\right) \\
& \Delta_{4}=\left[\Gamma_{1}^{2}\left(\Gamma_{1} \Gamma_{4}-\Gamma_{0} \Gamma_{3}\right)-\Gamma_{0}^{2}\left(\Gamma_{0} \Gamma_{2}+2 \Gamma_{1} \Gamma_{3}\right)\right] /\left(\Gamma_{0}+\Gamma_{1}\right)^{3}
\end{aligned}
$$

which are the same as those in equations (3.23) of [13]. Note that any restrictions on the form of the strain energy function $\Sigma$ have not been placed. The values of the above coefficients are given in [13] for some well-known materials. For example, in the case of Ko compressible material, these coefficients take the values $\Delta_{0}=1 / 3, \Delta_{1}=1, \Delta_{2}=-8, \Delta_{3}=4 / 3$, and $\Delta_{4}=-4 / 9$ where $T_{0}=\mu$ and $\mu$ is a material constant. Similarly, for the materially linear elastic solids, these coefficients take the values $\Delta_{0}=v /(1-v), \Delta_{1}=1$ and $\Delta_{2}=\Delta_{3}=\Delta_{4}=0$ where $T_{0}=\mu$, $v=\lambda / 2(\lambda+\mu)$ is Poisson's ratio and $\lambda$ and $\mu$ are Lamé constants. As is seen from equation (3.26) and (3.31), the coefficients $\Gamma_{2}, \Gamma_{3}$ and $\Gamma_{4}$ (and consequently $\Delta_{2}, \Delta_{3}$ and $\Delta_{4}$ ) show the effect of material non-linearity. Therefore it is deduced from the reduced constitutive equations (3.30) that material non-linearity is included only in the second order approximation.

\subsection{Plate equations for the first three orders}

It has been already expressed above that there exist the constraints ${\stackrel{(0)}{u_{3,3}}}_{=}=0, \stackrel{(0)}{u}_{\alpha, 3}=0$ and ${\stackrel{(1)}{u_{3,3}}}_{3}\left(2+{\stackrel{(1)}{u_{3,3}}}\right)=0$ on the displacement vector as a consequence of the expansion (3.2). From the first and the second constraints, the zeroth order displacement field is found as

$$
\stackrel{(0)}{u_{3}}=\stackrel{(0)}{w}\left(\xi^{1}, \xi^{2}\right), \quad \stackrel{(0)}{u_{\alpha}}=\stackrel{(0)}{v_{\alpha}}\left(\xi^{1}, \xi^{2}\right)
$$


which implies that the zeroth order displacement field is a membrane displacement field, i.e. it is free from the transverse coordinate $\zeta$ (henceforth $\zeta$ is used instead of $\xi^{3}$ for convenience). The third constraint expresses that $\stackrel{(1)}{u}_{3,3}=0$ or $\stackrel{(1)}{u}_{3,3}=-2$ should be satisfied. Now, as in [10], consider the unilateral constraint $j=\operatorname{det}\left(x_{, K}^{k}\right)>0$. If (3.1) is used in this constraint ensuring that the map $x^{k}=x^{k}\left(X^{K}\right)$ is orientation preserving, it takes the form $j=1+\stackrel{(1)}{u_{3,3}}+O(\delta)$. In other words, while $\delta$ goes to zero, the condition $\stackrel{(1)}{u_{3,3}}>-1$ must be satisfied which implies that ${\stackrel{(1)}{u_{3,3}}}=0$. Now, using (3.3), the restrictions imposed by the conditions (3.21) on the displacement vector are investigated. The condition $\stackrel{(0)}{E}_{\alpha 3}=0$ implies that $\stackrel{(1)}{u}_{\alpha, 3}=-\stackrel{(0)}{w}$. Thus the first order displacement field is obtained in the form

$$
\stackrel{(1)}{u_{3}}=\stackrel{(1)}{w}, \quad \stackrel{(1)}{u_{\alpha}}=\stackrel{(1)}{v_{\alpha}}-\zeta^{(0)} w_{, \alpha}
$$

where $\stackrel{(1)}{w}=\stackrel{(1)}{w}\left(\xi^{1}, \xi^{2}\right)$ and $\stackrel{(1)}{v_{\alpha}}=\stackrel{(1)}{v_{\alpha}}\left(\xi^{1}, \xi^{2}\right)$. Note that the first order displacement field includes the transverse coordinate $\zeta$ which does not appear in the zeroth order approximation. Similarly the conditions $\stackrel{(0)}{E}_{33}=0$ and $\stackrel{(1)}{E}_{\alpha 3}=0$ require that $\stackrel{(2)}{u}_{3,3}=0$ and $\stackrel{(2)}{u_{\alpha, 3}}=-\stackrel{(1)}{w}$, , respectively. Employing these relations, the second order displacement field is found as

$$
\stackrel{(2)}{u_{3}}=\stackrel{(2)}{w}, \quad \stackrel{(2)}{u_{\alpha}}=\stackrel{(2)}{v_{\alpha}}-\zeta^{(1)} w_{, \alpha}
$$

where $\stackrel{(2)}{w}=\stackrel{(2)}{w}\left(\xi^{1}, \xi^{2}\right)$ and $\stackrel{(2)}{v_{\alpha}}=\stackrel{(2)}{v_{\alpha}}\left(\xi^{1}, \xi^{2}\right)$. Since the lowest three order approximations only are considered here, the condition $\stackrel{(2)}{E_{\alpha 3}}=0$ which can be used to determine $\stackrel{(3)}{u_{\alpha}}$ will not be employed.

Noting that the quantities having the negative index are zero, for the sake of brevity, the constrained forms of the displacement field for the first three order approximations can be written altogether as $\stackrel{(k)}{u_{3}}=\stackrel{(k)}{w}$ and $\stackrel{(k)}{u_{\alpha}}=\stackrel{(k)}{v_{\alpha}}-\zeta^{(k-1)} w_{. \alpha}$ for $k=0,1,2$. In this case, using (3.3) the in-plane strain components can be expressed in the form $\dagger$

$$
\stackrel{(k)}{E}_{\alpha \beta}=\frac{1}{2} \stackrel{(k)}{\theta}_{\alpha \beta}-\zeta^{(k-1)} \stackrel{w}{w \alpha \beta}+\stackrel{(k-2)}{E}_{\alpha \beta}
$$

where $\stackrel{(k)}{\theta}_{\alpha \beta}$ and ${\stackrel{(0)}{\tilde{E}_{\alpha \beta}}}$ are defined by

$$
\stackrel{(k)}{\theta_{\alpha \beta}}\left(\xi^{1}, \xi^{2}\right)=\stackrel{(k)}{v}_{\alpha ; \beta}+\stackrel{(k)}{v_{\beta ; \alpha}}+\sum_{l=0}^{k} \stackrel{(l)}{w}_{, \alpha}{ }^{(k-l)}{ }^{w}, \quad \stackrel{(0)}{\tilde{E}_{\alpha \beta}}\left(\xi^{1}, \xi^{2}\right)=\frac{1}{2}{\stackrel{(0)}{v_{\gamma ; \alpha}}}^{(0)} v_{; \beta}^{\gamma}
$$

Note that $\stackrel{(0)}{E}_{\alpha \beta}$ is independent of $\zeta$ whereas $\stackrel{(1)}{E}_{\alpha \beta}$ and $\stackrel{(2)}{E}_{\alpha \beta}$ depend linearly on $\zeta$. Employing $(k)$

(3.35) in (3.30), the stress components $\sigma^{\alpha \beta}$ are written altogether as follows:

$$
\stackrel{(k)}{\sigma^{\alpha \beta}}=\Delta_{1}\left[\Delta_{0} \theta_{\gamma}^{\gamma} A^{\alpha \beta}+\stackrel{(k)}{\theta^{\alpha \beta}}-2 \zeta\left(\Delta_{0}{ }^{(k-1)} w_{; \gamma}^{\gamma} A^{\alpha \beta}+{ }^{(k-1)} w_{;}^{\alpha \beta}\right)\right]+{ }^{(k-2)} \tilde{\sigma}^{\alpha \beta}
$$

where ${\stackrel{(0)}{\tilde{\sigma}^{\alpha \beta}}}^{\alpha \beta}$ is obtained by substituting $\stackrel{(0)}{\tilde{E}}^{\alpha \beta}$ instead of $\stackrel{(2)}{E}^{\alpha \beta}$ in $(3.30)_{3}$ and is, in principle, a 
known function of the zeroth order terms. Recalling that $\stackrel{(0)}{\tilde{E}}^{\alpha \beta}$ and consequently $\stackrel{(0)}{\tilde{\sigma}^{\alpha \beta}}$ is independent of $\zeta$, it is seen from (3.37) that $\stackrel{(1)}{\sigma \beta}^{\alpha \beta}$ and $\stackrel{(2)}{\sigma^{\alpha \beta}}$ vary linearly along the thickness of (0)

the plate whereas $\sigma^{\alpha \beta}$ is uniform over the thickness. This case differs from that in [13] where the zeroth order stress components only depend linearly on $\zeta$ and this dependence is non-linear for higher order approximations.

Define the following zeroth and first order moments of the stress field:

$$
\stackrel{(k)}{n^{\alpha \beta}}=\int_{-1}^{1} \stackrel{(k)}{\sigma^{\alpha \beta}} \mathrm{d} \zeta, \quad \stackrel{(k)}{m^{\alpha \beta}}=\int_{-1}^{1} \stackrel{(k)}{\sigma^{\alpha \beta} \zeta} \mathrm{d} \zeta, \quad \stackrel{(k)}{q^{\alpha}}=\int_{-1}^{1} \stackrel{(k)}{\sigma^{\alpha 3}} \mathrm{~d} \zeta .
$$

Employing (3.37) in these definitions, it is found that

$$
\stackrel{(k)}{n^{\alpha \beta}}=2 \Delta_{1}\left(\Delta_{0} \stackrel{(k)}{\theta}_{\gamma}^{\gamma} A^{\alpha \beta}+\stackrel{(k)}{\theta^{\alpha \beta}}\right)+2 \stackrel{(k-2)}{\tilde{\sigma}^{\alpha \beta}}, \quad \stackrel{(k)}{m^{\alpha \beta}}=-\frac{4}{3} \Delta_{1}\left(\Delta_{0} \stackrel{(k-1)}{w_{; \gamma}^{\gamma}} A^{\alpha \beta}+\stackrel{(k-1)}{w_{;}^{\alpha \beta}}\right)
$$

(0)

Note that $m^{\alpha \beta}=0$, i.e. the bending moments are not included in the zeroth order approximation. Using equations (3.37) and (3.39), the in-plane stress components can be expressed as

$$
\sigma^{\alpha \beta}=\frac{1}{2}{ }^{(k)} n^{\alpha \beta}+\frac{3}{2} \zeta m^{(k)}
$$

This relation shows that, for the lowest three order approximations, the total stress can be decomposed into bending and membrane stress components. However, in [13], this property is valid only for the zeroth order approximation due to the reason expressed above.

In order to obtain the equilibrium equations for two-dimensional fields, (3.6) is integrated with respect to $\zeta$ between the limits -1 and 1 for $k=0,1,2$. Employing the definitions (3.38) and the boundary conditions (3.7) in the resulting expression, the following equations are found:

$$
\begin{aligned}
& \stackrel{(k)}{n_{; \alpha}^{\alpha \beta}}+\stackrel{(k)}{r^{\beta}}=0 \\
& \sum_{l=0}^{k}\left(^{(l)} n^{\alpha \beta}{ }^{(k-l)} w_{; \alpha \beta}-\stackrel{(l)}{r}^{\alpha}{ }^{(k-l)} w_{, \alpha}\right)+\stackrel{(k)}{p}=0
\end{aligned}
$$

where

$$
\begin{aligned}
& \stackrel{(0)}{r^{\beta}}\left(\xi^{1}, \xi^{2}\right)=g_{+}^{\beta}+g_{-}^{\beta}+\int_{-1}^{1} f^{\beta} \mathrm{d} \zeta, \quad \stackrel{(0)}{p}\left(\xi^{1}, \xi^{2}\right)=g_{+}^{3}+g_{-}^{3}+\int_{-1}^{1} f^{3} \mathrm{~d} \zeta \\
& \stackrel{(1)}{r^{\beta}}=0, \quad \stackrel{(1)}{p}\left(\xi^{1}, \xi^{2}\right)=\stackrel{(0)}{q_{; \alpha}^{\alpha}} \\
& \stackrel{(2)}{r^{\beta}}\left(\xi^{1}, \xi^{2}\right)=\stackrel{(0)}{\left(n^{\alpha \gamma} v_{; \gamma}^{\beta}\right)_{; \alpha},} \quad \stackrel{(2)}{p}\left(\xi^{1}, \xi^{2}\right)=\stackrel{(1)}{q_{; \alpha}^{\alpha} .}
\end{aligned}
$$

In order to calculate the shearing forces $\stackrel{(0)}{q^{\alpha}}$ and $\stackrel{(1)}{q^{\alpha}}$ appearing in these definitions, $(3.6)_{1}$ is multiplied by $\zeta$ (for $k=0,1$ ) and is integrated with respect to $\zeta$ in the interval $[-1,1]$ which gives

$$
\stackrel{(0)}{q^{\beta}}=g_{+}^{\beta}-g_{-}^{\beta}+\int_{-1}^{1} f^{\beta} \zeta \mathrm{d} \zeta, \quad \stackrel{(1)}{q^{\beta}}=\stackrel{(1)}{m_{; \alpha}^{\alpha \beta}}, \quad\left(\text { or } \stackrel{(1)}{q^{\beta}}=-\frac{4}{3} \Delta_{1}\left(1+\Delta_{0}\right) \stackrel{(0)}{w_{;}^{\alpha} \alpha^{\beta}}\right)
$$

Thus it is seen from (3.42) and (3.43) that, while $\stackrel{(0)}{r^{\beta}}, \stackrel{(0)}{p}$, and $\stackrel{(1)}{p}$ depend only on external loads, $\stackrel{(2)}{r}$ and $\stackrel{(2)}{p}$ are known functions of the zeroth order terms. 
Sometimes it may be more reasonable to express the moment equations (3.41) in terms of the displacements of the middle plane. In this case, (3.39) and (3.36) are employed in (3.41) and the following equations are obtained:

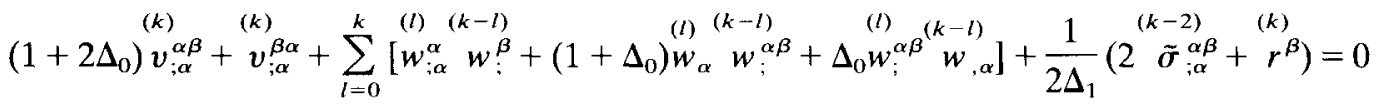

$$
\begin{aligned}
& \sum_{l=0}^{k}\left[\Delta_{0}\left(2 v_{; \gamma}^{\gamma}+\sum_{j=0}^{l} w_{;}^{(j)} w_{, \gamma}^{(l-j)}\right)^{(k-l)} w_{; \beta}^{\beta}+\left(2 v_{;}^{\alpha \beta}+\sum_{j=0}^{l} w_{;}^{(j)} w_{;}^{(l-j)}\right)^{(k-l)} w_{; \alpha \beta}\right] \\
& +\frac{1}{2 \Delta_{1}}\left[\sum_{l=0}^{k}\left(2 \tilde{\sigma}^{(l-2)} \tilde{w}_{; \alpha \beta}^{(k-l)}-\stackrel{(l)}{r}^{\gamma}{ }_{w, \gamma}^{(k-l)}\right)+\stackrel{(k)}{p}\right]=0 .
\end{aligned}
$$

These equations are non-linear for $k=0$ and are linear for $k=1,2$ as a consequence of the asymptotic expansion technique. As is known, problems in the plate theory may be solved by two methods-in terms of the displacements of the middle plane or in terms of the forces and moments. The first method employs (3.44) consisting of exactly three equations for three unknowns ${ }^{(k)} v^{\alpha}$ and $\stackrel{(k)}{w}$. Of course these equations must be completed with suitable boundary conditions. For example, it has been expressed above that the boundary conditions for clamped edge are in the form $\stackrel{(n)}{u_{\alpha}}=0$ and $\stackrel{(n)}{u_{3}}=0$ (for $n \geqslant 0$ ) on $\sigma$. Since $\stackrel{(n)}{u_{\alpha}}$ (for $n \geqslant 1$ ) and $\stackrel{(n)}{u_{3}}$ (for $n \geqslant 3$ ) are also functions of $\zeta$, the integration constants are less than those required by the boundary conditions. In order to remedy this situation two artifices may be used as in [13]. One method of approach is to prescribe the boundary conditions on averaged displacements and the other method is to prescribe the boundary conditions on the middle plane only, i.e. for $\zeta=0$. Here, for the lowest three order approximations, it can be easily seen that both of these approaches yield the same results which are $\stackrel{(k)}{v_{\alpha}}=0$ and $\stackrel{(k)}{w}=0$ on $\sigma$.

On the other hand, if the boundary conditions are imposed on the surface tractions, it may be more reasonable to choose ${ }^{(k)} n^{\alpha \beta}$ and $\stackrel{(k)}{w}$ as fundamental unknowns. But, in this case, as is seen from (3.41) there exist only three equations for the four unknowns. The other equation is provided by the compatibility condition which is obtained as follows. Using the definition $(3.36)_{1}$ it is easily found that

$$
\varepsilon_{\alpha \gamma} \varepsilon^{\beta \delta} \theta_{\beta ; \delta}^{(k)}=-\sum_{l=0}^{k}\left[\begin{array}{ll}
(l) \\
w
\end{array}, \stackrel{(k-l)}{w}\right]
$$

where $\varepsilon_{\alpha \beta}$ is a two-dimensional alternating tensor and the following notation is used:

$$
[\Phi, \Psi]=\Phi_{; \alpha}^{\alpha} \Psi_{; \beta}^{\beta}-\Phi_{;}^{\alpha \beta} \Psi_{; \alpha \beta}=\varepsilon^{\gamma \alpha} \varepsilon^{\delta \beta} \Phi_{; \gamma \delta} \Psi_{; \alpha \beta} .
$$

Here $\Phi$ and $\Psi$ are scalar functions of $\xi^{1}$ and $\xi^{2}$. If $\theta^{(k)}$ is calculated from (3.39) 1 as follows:

$$
\stackrel{(k)}{\theta^{\alpha \beta}}=\frac{1}{2 \Delta_{1}}\left[\stackrel{(k)}{n^{\alpha \beta}}-\frac{\Delta_{0}}{1+2 \Delta_{0}} \stackrel{(k)}{n}_{\gamma}^{\gamma} A^{\alpha \beta}-2\left(\stackrel{(k-2)}{\tilde{\sigma}^{\alpha \beta}-\frac{\Delta}{0}_{1+2 \Delta_{0}}} \stackrel{(k-2)}{\tilde{\sigma}_{\gamma}^{\gamma} A^{\alpha \beta}}\right)\right]
$$

and then this result is substituted into (3.45), the compatibility condition is obtained as

$$
\stackrel{(k)}{n_{; \alpha \beta}^{\alpha \beta}}-\frac{1+\Delta_{0}}{1+2 \Delta_{0}} \stackrel{(k)}{n_{\alpha ; \beta}^{\alpha \beta}}=2 \Delta_{1} \sum_{l=0}^{k}[\stackrel{(l)}{w}, \stackrel{(k-l)}{w}]+2\left(\stackrel{(k-2)}{\tilde{\sigma}_{; \alpha \beta}^{\alpha \beta}}-\frac{1+\Delta_{0}}{1+2 \Delta_{0}} \stackrel{(k-2)}{\tilde{\sigma}_{\alpha ; \beta}^{\alpha \beta}}\right)
$$

Thus there exist exactly four equations consisting of (3.41) and (3.48) for the four unknowns 

$n^{\alpha \beta}$ and ${ }^{(k)} w$ in a stress type formulation. In this case, integrating $(3.8)_{1}$ with respect to $\zeta$ on $[-1,1]$, the boundary conditions on ${ }^{(k)}{ }^{\alpha \beta}$ are found in the form

$$
\stackrel{(k)}{n^{\alpha \beta}} n_{\alpha}=\stackrel{(k)}{h}{ }^{\beta} \text { on } c
$$

where

$$
\stackrel{(0)}{h^{\beta}}=\int_{-1}^{1} \tau^{\beta} \mathrm{d} \zeta, \quad \stackrel{(1)}{h^{\beta}}=0, \quad \stackrel{(2)}{h^{\beta}}=-\left(\stackrel{(0)}{n^{\alpha \gamma}} v_{; \gamma}^{(0)}\right) n_{\alpha}
$$

In order to express the system (3.41) and (3.48) in a simpler form, the solution of $(3.41)_{1}$ is written as follows:

$$
\stackrel{(k)}{n^{\alpha \beta}}=\stackrel{(k)}{\bar{n}^{\alpha \beta}}+\stackrel{(k)}{\eta^{\alpha \beta}}
$$

where $\bar{n}^{\alpha \beta}$ and $\stackrel{(k)}{\eta^{\alpha \beta}}$ satisfy the following relations:

$$
\stackrel{(k)}{\bar{n}_{; \alpha}^{\alpha \beta}}=0, \quad \stackrel{(k)}{\eta_{; \alpha}^{\alpha \beta}}=-\stackrel{(k)}{r^{\beta}} .
$$

Obviously, (3.52) $)_{1}$ implies that there exists a stress function $\stackrel{(k)}{\Phi}\left(\xi^{1}, \xi^{2}\right)$ and $\stackrel{(k)}{\bar{n}^{\alpha \beta}}$ can be expressed as

$$
\stackrel{(k)}{n}^{\alpha \beta}=\varepsilon^{\gamma \alpha} \varepsilon^{\delta \beta} \stackrel{(k)}{\Phi}_{; \gamma \delta}
$$

which satisfies $(3.52)_{1}$ identically. In order to reach the equations necessary to determine $\stackrel{(k)}{\Phi}$ and $\stackrel{(k)}{w}$, the above definitions are introduced into $(3.41)_{2}$ and $(3.48)$ and the following coupled equations are found:

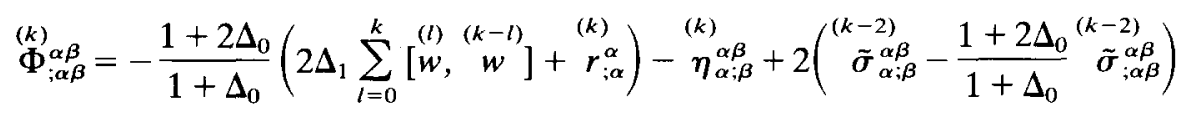

$$
\begin{aligned}
& \sum_{l=0}^{k}\left([\stackrel{(l)}{\Phi}, \stackrel{(k-l)}{w}]+\stackrel{(l)}{\alpha \beta}^{(k-l)} w_{; \alpha \beta}-{\stackrel{(l)}{r^{\alpha}}}^{(k-l)} w_{, \alpha}\right)+\stackrel{(k)}{p}=0 .
\end{aligned}
$$

For $k=0$ these equations correspond to a generalized form of the well-known non-linear Föppl membrane equations [7,16] and the original equations can be obtained by neglecting the body forces and the surface tractions $g_{\mp}^{\alpha}$ prescribed on the plate faces. Note that the equations (3.54) are linear for $k=1$, 2. If the above definitions are used in (3.49), the boundary conditions on $\stackrel{(k)}{\Phi}$ are found in the form

$$
\varepsilon^{\gamma \alpha} \varepsilon^{\delta \beta^{(k)}} \stackrel{(k \gamma \delta}{h}_{n_{\alpha}}=\stackrel{(k)}{h^{\beta}}-\stackrel{(k)}{\eta}^{\alpha \beta} n_{\alpha} \quad \text { on } c
$$

\subsection{Incompressible solids}

In the remainder of this section, how the asymptotic expansion method presented above should be modified for incompressible hyperelastic materials is discussed. As it is known, the constraint of incompressibility, ensuring that every possible material deformation is isochoric, 
introduces a scalar multiplier interpreted as a pressure into the constitutive equations. Therefore, the fundamental difference in applying the asymptotic expansion method to the compressible and incompressible hyperelastic plates arises from the difference between the constitutive equations. The approach presented in [14] can be followed mainly, where an asymptotic theory leading to the von Kármán equations in the zeroth order approximation is constructed for incompressible materials. In that approach, firstly, expanding the pressure function in asymptotic series, a hierarchy of the constitutive equations in which the strain energy function is an arbitrary function of the first two invariants of the Green deformation tensor is obtained. Later, the incompressibility condition is used to eliminate the pressure terms from the hierarchy of the constitutive equations. Consequently, it is shown that the results obtained for compressible solids are also valid for the incompressible hyperelastic plates with the use of a transformation between the material coefficients. Following the same approach (for the sake of brevity, the detailed calculations are not presented here), it is deduced that the same transformation is also valid for the present non-linear membrane theory. In other words, the above equations obtained for the case of compressible solids are also valid for the incompressible plates under the transformation

$$
\Delta_{0} \rightarrow 1, \quad \Delta_{1} \rightarrow \Lambda_{0}, \quad \Delta_{2} \rightarrow 4\left(\Lambda_{1}-\Lambda_{0}\right), \quad \Delta_{3} \rightarrow 4 \Lambda_{0}, \quad \Delta_{4} \rightarrow-4\left(\Lambda_{0}+\Lambda_{1}\right)
$$

where the following definitions are used:

$$
\begin{aligned}
& \Lambda_{0}=\left.2\left(\frac{\partial \Sigma}{\partial I_{C}}+\frac{\partial \Sigma}{\partial I I_{C}}\right)\right|_{0}, \quad \Lambda_{1}=-\left.2 \frac{\partial \Sigma}{\partial I_{C}}\right|_{0}, \\
& \Lambda_{2}=\left.2\left(\frac{\partial^{2} \Sigma}{\partial I_{C}^{2}}+3 \frac{\partial^{2} \Sigma}{\partial I_{C} \partial I_{C}}+2 \frac{\partial^{2} \Sigma}{\partial I I_{C}^{2}}+\frac{\partial \Sigma}{\partial I I_{C}}\right)\right|_{0}
\end{aligned}
$$

which are the same as those in equations (3.13) of [14]. Here $I_{C}$ and $I I_{C}$ are the first and second invariants of the Green deformation tensor, respectively and the subscript 0 represents the case where the quantities are evaluated at $I_{C}=I I_{C}=3$. The values of these coefficients are given in [14] for some well-known materials, for example, $\Lambda_{0}=2$ and $\Lambda_{1}=\Lambda_{2}=0$ for the neo-Hookean incompressible materials.

\section{THE PROBLEM OF AN INFINITE STRIP UNDERGOING LARGE TRANSVERSE DEFLECTION}

\subsection{Description of the problem}

In this section the problem of an infinite strip subject to uniform load, undergoing large transverse deflection under clamped edge conditions is considered. The reason for choosing this relatively easy problem is that it has been investigated in [14] and therefore the results of these two approximations can be compared. Since the method of solution is the same as that in [14], the results only are given here.

Assume that a homogeneous, isotropic and hyperelastic infinite strip occupies the region $-\infty \leqslant X_{1} \leqslant \infty,-L \leqslant X_{2} \leqslant L$ and $-h \leqslant X_{3} \leqslant h$ in $\mathbf{X}$-Cartesian coordinates and that the upper face of the strip is subject to uniform load $q_{0}$. Moreover, the body forces are neglected and the deformation is assumed to be as follows:

$$
u_{1}=0, \quad u_{2}=u_{2}\left(\xi_{2}, \zeta\right), \quad u_{3}=u_{3}\left(\xi_{2}, \zeta\right)
$$

with $X_{2}=L \xi_{2}, X_{3}=\varepsilon L \zeta$ and $\varepsilon=h / L$. From these assumptions, it follows that

$$
f_{\alpha}=f_{3}=g_{\alpha}^{\mp}=g_{3}^{-}=\tau_{\alpha}=\tau_{3}=\stackrel{(0)}{r_{\alpha}}=0, \quad \stackrel{(0)}{p}=g_{3}^{+}=p_{0}
$$


where $p_{0}=\bar{q}_{o} / \varepsilon^{5 / 2}$ and $\bar{q}_{0}=q_{0} / T_{0}$. Recalling the boundary conditions expressed for clamped edges, the boundary conditions for the present problem are written as

$$
\stackrel{(k)}{v_{2}}=0, \quad \stackrel{(k)}{w}=0 \quad \text { at } \quad \xi_{2}=\mp 1
$$

for $k=0,1,2$. Using an approximation which is similar to that in [14], the second-order ordinary differential equations obtained from (3.44) for this special case are solved analytically under the above boundary conditions.

\subsection{Zeroth order approximation}

The displacement field:

$$
\stackrel{(0)}{w}=\frac{1}{2}(6 \gamma)^{1 / 3}\left(1-\xi_{2}^{2}\right), \quad \stackrel{(0)}{v_{2}}=\left(\frac{\gamma^{2}}{6}\right)^{1 / 3}\left(\xi_{2}-\xi_{2}^{3}\right)
$$

where the load parameter $\gamma$ is defined by $\gamma=p_{0} / 4 \Delta_{1}\left(1+\Delta_{0}\right)$.

The stress and moment components:

$$
\stackrel{(0)}{\sigma_{22}}=2 \Delta_{1}\left(1+\Delta_{0}\right)\left(\frac{\gamma^{2}}{6}\right)^{1 / 3}, \quad \stackrel{(0)}{n}_{22}=2{\stackrel{(0)}{\sigma_{22}},}^{m_{22}}=0
$$

\subsection{First order approximation}

The displacement field:

$$
\stackrel{(1)}{w}=\stackrel{(1)}{v_{2}}=0 \text {. }
$$

The stress and moment components:

$$
\stackrel{(1)}{\sigma_{22}}=2 \Delta_{1}\left(1+\Delta_{0}\right)(6 \gamma)^{1 / 3} \zeta, \quad \stackrel{(1)}{n_{22}}=0, \quad \stackrel{(1)}{m_{22}}=\frac{4}{3} \Delta_{1}\left(1+\Delta_{0}\right)(6 \gamma)^{1 / 3}
$$

\subsection{Second order approximation}

The displacement field:

$$
\begin{aligned}
& \stackrel{(2)}{w}=-\frac{\gamma}{60}\left[\left(5 \Delta_{N}-42\right)\left(1-\xi_{2}^{2}\right)+45\left(1-\xi_{2}^{4}\right)\right] \\
& \stackrel{(2)}{v_{2}}=-\frac{1}{30}\left(\frac{\gamma^{2}}{6}\right)^{2 / 3}\left[10 \Delta_{N}\left(\xi_{2}-\xi_{2}^{3}\right)+3\left(19 \xi_{2}+8 \xi_{2}^{3}-27 \xi_{2}^{5}\right)\right]
\end{aligned}
$$

where the parameter $\Delta_{N}$ measuring the effect of material non-linearity is defined as $\Delta_{N}=\left(\Delta_{2}+\Delta_{4}-\Delta_{3}\right) / \Delta_{1}\left(1+\Delta_{0}\right)$.

The stress and moment components:

$$
\stackrel{(2)}{\sigma_{22}}=\frac{1}{15} \Delta_{1}\left(1+\Delta_{0}\right)\left(\frac{\gamma^{2}}{6}\right)^{2 / 3}\left(5 \Delta_{N}-42+90 \xi_{2}^{2}\right), \quad \stackrel{(2)}{n_{22}}=2 \stackrel{(2)}{\sigma_{22}}, \quad \stackrel{(2)}{m_{22}}=0
$$

\subsection{Numerical results}

Now the results obtained for the lowest three order approximations are investigated numerically from two points of view for both compressible and incompressible materials. The first point is to compare the solutions corresponding to material and geometrical nonlinearities. Note that, in order to discard material non-linearity by keeping geometric non-linearity, it suffices only to substitute $\Delta_{0}=v /(1-v), \Delta_{1}=1$ and $\Delta_{N}=0$ into the above relations. The second point is to compare the results obtained here with those in [14], which 
are obtained as a consequence of the asymptotic expansion giving the well-known von Kármán plate equations in the zeroth order approximation.

Firstly, load-maximum deflection curves corresponding to a strip made of the Ko compressible material for which $\Delta_{0}=1 / 3, \Delta_{1}=1$ and $\Delta_{N}=-22 / 3$ are depicted in Fig. 1(a) and (b) for $\varepsilon=0.01$ and $\varepsilon=0.005$, respectively. In these figures, the curves corresponding to the
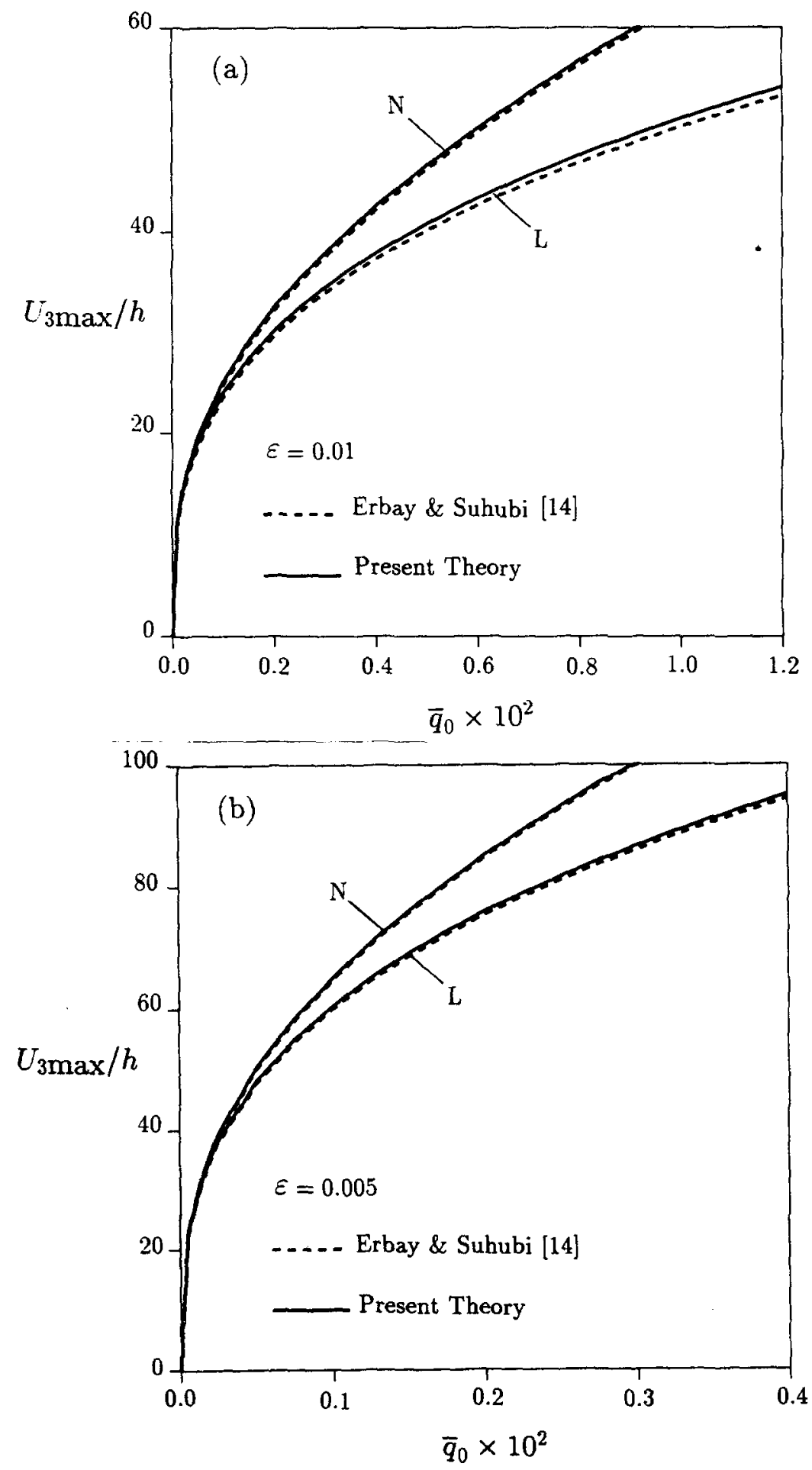

Fig. 1. Load versus maximum deflection curves for a clamped strip made of the Ko compressible material for (a) $\varepsilon=0.01$ and (b) $\varepsilon=0.005$. L and $N$ show the cases where $\Delta_{N}=0$ and $\Delta_{N} \neq 0$, respectively. 


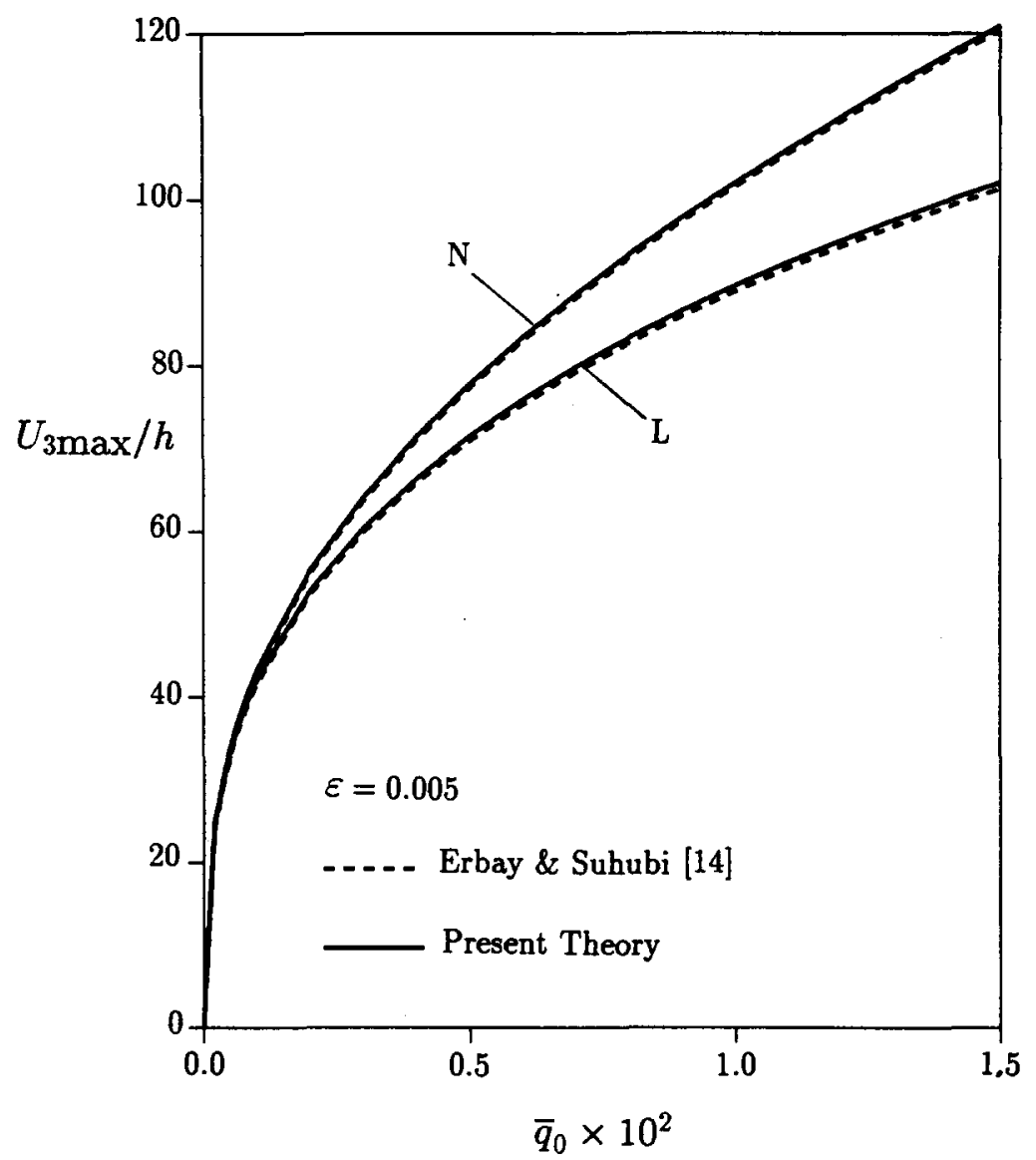

Fig. 2. Load versus maximum deflection curves for a clamped strip made of the neo-Hookean incompressible material for $\varepsilon=0.005$. $\mathrm{L}$ and $\mathrm{N}$ show the cases where $\Delta_{N}=0$ and $\Delta_{N} \neq 0$, respectively.

materially linear case (i.e. $\Delta_{N}=0$ ) and the materially non-linear case (i.e. $\Delta_{N} \neq 0$ ) are shown by the letters $\mathrm{L}$ and $\mathrm{N}$, respectively. While the broken line is used to show the results in [14], the solid line shows the results of the present work. Moreover, the maximum deflection is defined as $U_{3 \max }=\left.\varepsilon^{1 / 2} L\left(\stackrel{(0)}{w}+\varepsilon^{1 / 2} w+\varepsilon w\right)\right|_{\xi_{2}=0}$. As is seen from these figures, there is a very close conformity between the results corresponding to the present theory and to [14]. This situation seems reasonable recalling that for very large deflections the membrane effects dominate everywhere except the region near the edge [14]. Generally, the remarks made in [14] are also valid for the present work. For example, the larger the load or the thinner the plate, the greater is the difference between the maximum deflections corresponding to material and geometrical non-linearities. The approximate values given in [14] for the maximum deflections at which this difference is in the order of thickness are also valid here, roughly.

A similar graph is provided in Fig. 2 for a strip made of the neo-Hookean incompressible material for which $\Delta_{0}=1, \Delta_{1}=2$ and $\Delta_{N}=-6$. As is seen from the figure, the remarks made above for Fig. 1 are qualitatively valid for the present case too, in conformity with those in [14] for the neo-Hookean solids.

The variations of the axial force and the bending moment along the axis of a strip made of the Ko compressible material are shown in Fig. 3(a) and (b), respectively, for the deflections where the material non-linearity becomes significant. In these figures, the definitions $n_{22}=$ $\varepsilon\left(\stackrel{(0)}{n_{22}}+\varepsilon^{1 / 2} \stackrel{(1)}{n}_{22}+\varepsilon \stackrel{(2)}{\varepsilon}_{22}\right)$ and $m_{22}=\varepsilon\left(\stackrel{(0)}{m}_{22}+\varepsilon^{1 / 2} m_{22}^{(1)}+\varepsilon m_{22}^{(2)}\right)$ are used. It is obvious from these figures that the results of the present work are qualitatively similar to those given in [14] except the region near the edge. For example, as is also pointed out in [15], the membrane IJES 35-1-0 

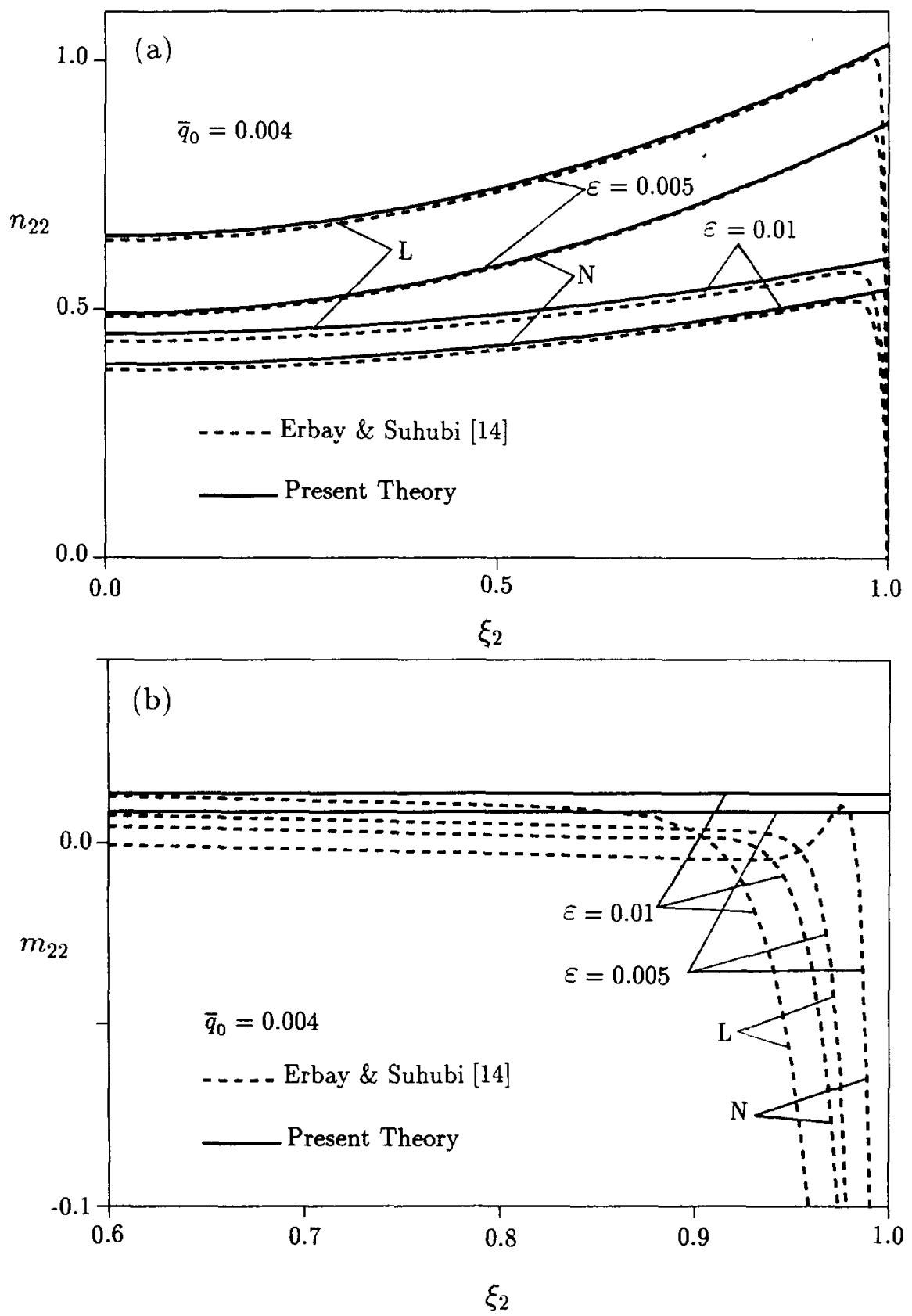

Fig. 3. Variations of (a) axial force and (b) bending moment along the length of the strip for a clamped strip made of the Ko compressible material. $\mathbf{L}$ and $N$ show the cases where $\Delta_{N}=0$ and $\Delta_{N} \neq 0$, respectively.

stresses are further increased by the thinning of the plate. Moreover, the larger the deflections (or the thinner the plate) the greater is the difference between the axial forces corresponding to material and geometrical non-linearities. However, for the lowest three order approximations in the present work, the bending moments corresponding to material and geometrical non-linearities are the same, i.e. it is a constant and independent of $\Delta_{N}$. On the other hand, as is also pointed out in [15], the axial force increases significantly towards the plate's outer edge as a result of rotational effect. Clearly, near the plate edge, the values given by the present work and by [14] for both the axial force and the bending moment are very different. Whereas the axial force and the bending moment for the membrane approach presented in this work change uniformly along the axis, the axial force and the bending moment corresponding to [14] 


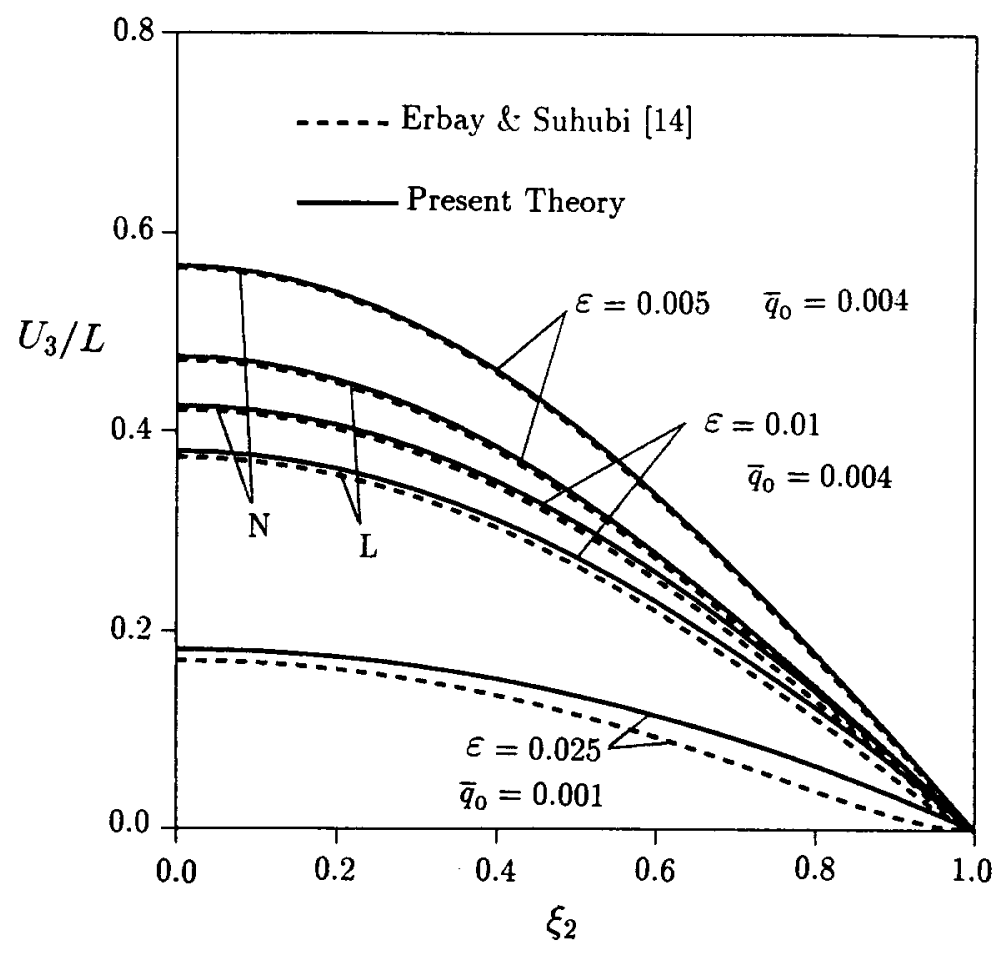

Fig. 4. Deformed profiles of a clamped strip made of the Ko compressible material. $L$ and $N$ show the cases where $\Delta_{N}=0$ and $\Delta_{N} \neq 0$, respectively.

change rapidly near the edge. The presence of the bending component of the boundary layer in a clamped plate has been known for many years [18] and it can also develop when the strains are small. Whereas this component has received considerable attention in the past [18-20], the existence of a membrane component of the boundary layer, which develops as the strains grow large, has first been indicated in [21] and [22]. Also the results of the present work and of [14] verify the existence of such a membrane component of the boundary layer forming when the strains grow large.

Finally, in Fig. 4, the deformed profiles of a strip made of the Ko compressible material are given for different loads and thickness parameters. As is expected, the larger the deflections, the smaller is the difference between the results of the present work and those of [14], and the greater is the difference between the results corresponding to material and geometrical non-linearities.

\section{CONCLUSIONS}

In this work, an asymptotic theory for membrane equations of thin hyperelastic plates is developed and the lowest three order equations of the asymptotic expansion are studied in detail. It is shown that the zeroth order approximation corresponds to the well known Föppl non-linear membrane equations, the first order approximation includes stress couples and the effect of material non-linearity becomes significant only in the second order approximation. It is also demonstrated that the first and second order in-plane stress components vary linearly along the thickness of the plate, whereas the zeroth order in-plane stress component is uniform over the thickness. Consequently, the stress can be decomposed into bending and membrane stress components for the lowest three order approximations. This case differs from that in [13] where the zeroth order stress components only depend linearly on the transverse coordinate and this dependence is non-linear for higher order approximations. Finally, the effect of material non-linearity is discussed numerically through the problem of an infinite strip subject 
to uniform load. Generally, the results obtained in [14] are also valid for the present case except the region near the edge. For instance, the values given for the maximum deflections where the material non-linearity becomes significant are almost the same (Figs 1 and 2). However, the values given by the present work and by [14] for both the axial force and the bending moment are very different near the plate edge.

As is expected, the forces and deformations obtained by the use of membrane theory usually become incompatible at the plate's boundary with the actual boundary conditions. Therefore the approximation presented here must be considered as an outer solution of the actual boundary value problem and it must be supplemented by a boundary layer solution that becomes insignificant away from the edge. The complete analysis requires an algorithm for computing the boundary layers, which becomes more complicated as we proceed to higher order approximations. A study of such an algorithm and the applicability of the present approach to the more popular loads and geometries will form a topic for further research.

Acknowledgement-The author gratefully acknowledges the partial support provided by Turkish Scientific and Technical Research Council through the project TBAG-U/14-1.

\section{REFERENCES}

[1] Habib, L. M., Int. J. Solids Structures, 1967, 3, 207.

[2] Ciarlet, P. G. and Destuynder, P., Comp. Meth. Appl. Mech. Engng, 1979, 17/18, 227.

[3] Ciarlet, P. G., Arch. Rational Mech. Anal., 1980, 73, 349.

[4] Suhubi, E. S., Bull. Tech. Univ. Istanbul, 1982, 35, 217.

[5] Coutris, N. and Monavon, A., Int. J. Engng Sci., 1988, 26, 1189.

[6] Berg, L. J., Int. J. Solids Structures, 1991, 27, 1401.

[7] Stoker, J. J., Nonlinear Elasticity. Gordon \& Breach, 1968.

[8] Johnson, M. W., Jr., and Urbanik, T. J., ASME J. Appl. Mech., 1984, 51, 146.

[9] Johnson, M. W., Jr., Int. J. Solids Structures, 1985, 21, 11.

[10] Davet, J. L., Modelisation Mathématique et Analyse Numérique, 1986, 20, 225.

[11] Karwowski, A., J. Elasticity, 1993, 32, 93.

[12] Fox, D. D., Raoult, A. and Simo, J. C., Arch. Rational Mech. Anal., 1993, 124, 157.

[13] Erbay, H. A. and Suhubi, E. S., Int. J. Engng Sci., 1991, 29, 447.

[14] Erbay, H. A. and Suhubi, E. S., Int. J. Engng Sci., 1991, 29, 467.

[15] Brodland, G. W., Int. J. Solids Structures, 1988, 24, 351.

[16] Föppl, A., Vorlesungen ïber Technische Mechanik, Vol. 5. R. Oldenbourg, 1907.

[17] Eringen, A. C., Nonlinear Theory of Continuous Media. McGraw-Hill, New York, 1962.

[18] Bromberg, E., Commun. Pure Appl. Math., 1956, 9, 633.

[19] Taber, L. A., ASME J. Appl. Mech., 1985, 52, 907.

[20] Brodland, G. W., Solid Mech. Arch., 1986, 11, 219.

[21] Taber, L. A., Int. J. Solids Structures, 1987, 23, 719.

[22] Taber, L. A., ASME J. Appl. Mech., 1987, 54, 280. 